Pacific Journal of Mathematics

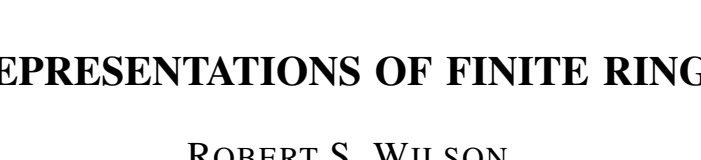




\title{
REPRESENTATIONS OF FINITE RINGS
}

\author{
ROBERT S. WILSON
}

\begin{abstract}
In this paper we extend the concept of the Szele representation of finite rings from the case where the coefficient ring is a cyclic ring to the case where it is a Galois ring. We then characterize completely primary and nilpotent finite rings as those rings whose Szele representations satisfy certain conditions.
\end{abstract}

1. Preliminaries. We first note that any finite ring is a direct sum of rings of prime power order. This follows from noticing that when one decomposes the additive group of a finite ring into its pime power components, the component subgroups are, in fact, ideals. So without loss of generality, up to direct sum formation, one needs only to consider rings of prime power order. For the remainder of this paper $p$ will denote an arbitrary, fixed prime and all rings will be of order $p^{n}$ for some positive integer $n$. Of the two classes of rings that will be studied in this paper, completely primary finite rings are always of prime power order, so for the completely primary case, there is no loss of generality at all. However, nilpotent finite rings do not need to have prime power order, but we need only classify finite nilpotent rings of prime power order, the general case following from direct sum formation.

If $R$ is finite ring (of order $p^{n}$ ) then the characteristic of $R$ will be $p^{k}$ for some positive integer $k$. If $x \in R$ then we define the order of $x$ to be the smallest positive integer $e$ such that $p^{e} x=0$. Thus $0<e \leqq k$.

We now define a very important class of finite rings.

DeFINITION 1.1. Let $f(x) \in Z[x]$, when $Z$ denotes the rational integers, be monic of degree $r$ and irreducible modulo $p$. Then the ring $Z[x] /\left(p^{k}, f(x)\right)$ is called the Galois ring of order $p^{k r}$ and characteristic $p^{k}$, and will be denoted by $G_{k, r}$. Basically, then, a Galois ring is an irreducible algebraic extension of degree $r$ of the cyclic ring $Z /\left(p^{k}\right)$, and any two irreducible algebraic extensions of $Z /\left(p^{k}\right)$ of degree $r$ are isomorphic $[2, \S 3]$. Note that $G_{1, r} \cong G F\left(p^{r}\right)$ and $G_{k, 1} \cong$ $Z /\left(p^{k}\right)$. This class of rings was introduced independently by Raghavendran [2] and Janusz [1] both of whom called them Galois rings. The importance of Galois rings, at least in our case, is that if $R$ is a completely primary finite ring of characteristic $p^{k}$ with Jacobson radical $J$ such that $R / J \cong G F\left(p^{r}\right)$ then $R$ contains a unique (up to inner isomorphism) copy of $G_{k, r}$ ([2, Th. 8]). Thus the com- 
pletely primary finite ring $R$ is a $G_{k, r}$-bimodule. The author has developed a structure theory for finite $G_{k, r}$-bimodules and facts that we will need are listed below.

Proposition 1.2. Let $M$ be a finite $G_{k, r}$-bimodule. Then there exist elements $m_{1}, \cdots, m_{n} \in M$ such that

(i) $\quad M=\sum_{i=1}^{n} \oplus G_{k, r} m_{i}$

(ii) $G_{k, r} m_{i}=m_{i} G_{k, r} i=1, \cdots, n$. Moreover, if $M=\sum_{j=1}^{q} \oplus G_{k, r} m_{j}^{\prime}$ is another such decomposition of $M$ then $q=n$ and the orders of the $m_{j}^{\prime}$ are (after possible reindexing) the orders of the $m_{i}$.

This is essentially Theorem 2.1 of [5].

2. The Szele representation. The representations we are interested in will be right regular representations with Galois rings as coefficient rings. Thus we adopt the convention of writing maps on the right and scalars on the left. We next introduce the following class of rings. Let $k=k_{1} \geqq k_{2} \geqq \cdots k_{n}>0$ be a nonincreasing sequence of positive integers. Let $\phi_{j}: G_{k, r} \rightarrow G_{k_{j}, r}$ be the map induced by the cannonical homomorphism $G_{k, r} \rightarrow G_{k, r} / p^{k_{j}} G_{k, r}$ followed by the isomorphism $G_{k, r} / p^{k_{j}} G_{k, r} \cong G_{k_{1}, r}$. Let $R$ denote the set of all rectangular arrays $\left[a_{i j}\right]$ where $a_{i j} \in G_{k_{j}, r}(i, j=1, \cdots, n)$ where for entries below the main diagonal we have $a_{i j}$ is a multiple of $p^{k_{j}-k_{i}}$ in $G_{k_{r}, r}$. Let $S=\left\{\left[b_{i j}\right] \in M_{n}\left(G_{k, r}\right) \mid b_{i j} \in p^{k_{j}-k_{i}} G_{k, r}\right.$ if $\left.i>j\right\}$. Define a map $\Phi: S \rightarrow R$ by $\Phi:\left[b_{i j}\right] \rightarrow\left[\left(b_{\imath j}\right) \phi_{j}\right]$. Clearly $\Phi$ is onto. We define addition and multiplication in $R$ by stipulating that $\Phi$ preserves addition and multiplication. $R$ is then a ring. The only thing which is not immediate is that multiplication is well-defined. But that follows from our condition that $a_{i j}$ is a multiple of $p^{k_{j}-k_{i}}$ whenever $i>j$. This construction is due to Szele [3] who did it for cyclic rings, however, as we shall see there is no reason why we cannot do it over more general rings. We shall call such a ring and subrings thereof rings of Szele matrices. The class of rings are of interest when studying finite rings because of the following result.

LEMMA 2.1. Let $R$ be a finite ring of characteristic $p^{k}$ which contains a copy of $G_{k, r}$. Then the right regular representation of $R$ over $G_{k, r}$ can be realized as a ring of Szele matrices.

\section{Thus we have immediately}

Corollary 2.2. Any finite ring with unit (of prime power of order) is a subring of a ring of Szele matrices over a Galois ring. 
These results were proved by Szele in case the coefficient Galois ring is taken to be a cyclic ring. Any finite ring will of course contain a cyclic ring and it is quite possible that the cyclic subring will be the largest Galois subring so, in a sense, our result does not represent much of a step forward in the study of general finite rings. However, in the study of completely primary finite rings, one can obtain a tractable, complete characterization if one studies the Szele representation over the largest Galois subring.

Proof of Lemma 2.1. Proposition 1.2 supplies all of the necessary tools to follows Szele's original proof except that, in general, Galois subrings do not need to be contained in the center. However, we circumvent that difficulty be writing maps on the right and scalars on the left. $G_{k, r} \subset R$ so $R$ is a $G_{k, r}$-bimodule. Let $b_{1}, \cdots, b_{n}$ be a basis of $R$ over $G_{k, r}$ satisfying the conditions of Proposition 1.2. Let $k_{i}$ be the order of $b_{i}$ and suppose that the $b_{i}$ are arranged such that $k=k_{1} \geqq k_{2} \geqq \cdots \geqq k_{n}>0$. Let $a \in R, b_{i} a \in R i=1, \cdots, n$ so we may write

$$
b_{i} \alpha=\sum_{j=1}^{n} \alpha_{i j} b_{j} \alpha_{i j} \in G_{k, r} i=1, \cdots, n .
$$

Note that

$$
0=p^{k_{i}} b_{i} a=\sum_{j=1}^{n} p^{k_{i}} \alpha_{i j} b_{j} \text { for all } i=1, \cdots, n .
$$

Since the $b_{j}$ 's are independent over $G_{k, r}$ we conclude that $p^{k_{i}} \alpha_{i j} b_{j}=0$ for all $i, j=1, \cdots, n$. Thus $p^{k_{i}} \alpha_{i j}$ is a multiple of $p^{k_{j}}$. So if $i>j$ we have that $\alpha_{i j}$ is a multiple of $p^{k_{j}-k_{i}}$ for all $a \in R$. So the map $\psi$ given by $\psi: a \rightarrow\left[\left(\alpha_{i j}\right) \phi_{j}\right]$ is a map from $R$ into a ring of Szele matrices. It is straightforward to check that $\psi$ is a ring homomorphism and since $k_{j}$ is the order of $b_{i}$ it follows that $\psi$ is one to one, and the lemma is proved.

3. Completely primary and nilpotent finite rings. In [5], the author proves that a completely primary (resp. nilpotent) finite ring is isomorphic to a subring of a homomorphic image of a ring of matrices which are upper triangular (resp. strictly upper triangular) modulo $p$. We now characterize this homomorphic image by means of the Szele representation over Galois rings.

THEOREM 3.1. Let $R$ be a completely primary finite ring of characteristic $p^{k}$ with radical $J$ such that $R / J \cong G F\left(p^{r}\right)$. Then $R$ is isomorphic to a ring of Szele matrices over $G_{k, r}$ in which every matrix is upper triangular modulo $p$, and if the matrix $\left[\left(a_{i j}\right) \phi_{j}\right]$ 
$\left(a_{i j} \in G_{k, r}\right)$ is in this ring then there are automorphisms $\sigma_{2}, \cdots, \sigma_{n}$ of $G F\left(p^{r}\right)$ such that $\left(a_{j j}\right) \phi_{1}=\left(\left(a_{11}\right) \dot{\phi}_{1}\right) \sigma_{j}$ (i.e., the main diagonal entries of the matrices are all related by automorphisms modulo p).

The converse is also true. Any such ring is completely primary, so this result characterizes completely primary finite rings.

Proof. We first note that if a completely primary ring has an representation as a ring of Szele matrices which is upper triangular modulo $p$ then the main diagonal entries must be related by automorphisms modulo $p$. For then the radical of the ring is the set of all matrices in the ring which are strictly upper triangular modulo $p$ so the map $R \rightarrow R / J \cong G F\left(p^{r}\right)$ can be realized by the map

$$
\left[\begin{array}{ccc}
\left(a_{11}\right) \dot{\phi}_{k} & \cdots & \left(a_{1 n}\right) \phi_{k_{n}} \\
\vdots & & \vdots \\
\left(a_{n 1}\right) \dot{\phi}_{k} & \cdots & \left(a_{n n}\right) \phi_{k_{n}}
\end{array}\right] \longrightarrow\left(\left(a_{11}\right) \dot{\phi}_{1}, \cdots,\left(a_{n n}\right) \phi_{1}\right) \in G F\left(p^{r}\right) \oplus \cdots \oplus G F\left(p^{r}\right)
$$

However, the image of this map is a one dimensional algebra over $G F\left(p^{r}\right)$ so the entry $\left(a_{j j}\right) \phi_{1}$ is uniquely determined by the value of $\left(a_{11}\right) \phi_{1}$; i.e., $\left(a_{j j}\right) \dot{\phi}_{1}$ is a function of $\left(a_{11}\right) \dot{\phi}_{1}$ say $\left(a_{j j}\right) \dot{\phi}_{1}=\left(\left(a_{11}\right) \dot{\phi}_{1}\right) \sigma_{j}$ for some function $\sigma_{j} j=2, \cdots, n$. The $\sigma_{j}$ are seen to be homomorphisms from $G F\left(p^{r}\right)$ to $G F\left(p^{r}\right)$ and that part of the result follows. For the rest of the result, the particular matrix representation we choose depends upon the choice of independent generating set we make for $R$ over $G_{k, r}$. For $R$ completely primary as in the hypothesis of the theorem, the author showed [5, Prop. 2.2] that in addition to conditions (i) and (ii) of Proposition 1.2 we can also assume (iii) $b_{1}=1$, and $b_{2}, \cdots, b_{n} \in J$.

We now obtain the correct independent generating set of $R$ over $G_{k, r}$. Let $e$ be the smallest positive integer such that $J^{e}=(0)$. Let us consider the set of independent generating sets of $R$ which satisfy the conditions (i), (ii), and (iii). Suppose that $q_{1}$ is the maximum number of elements of any of these generating sets which are in $J^{e-1}$. Say $\left\{1, b_{2}, \cdots, b_{q_{1}+1}, c_{q_{1}+2}, \cdots, c_{n}\right\}$ is such a generating set with $b_{2}, \cdots, b_{q_{1}+1} \in J^{e-1}$. Suppose $q_{2}$ is the maximum number of elements in $J^{e-2}$ included in any set of the form $\left\{1, b_{2}, \cdots, b_{q_{1}+1}, c_{q_{1}+2}, \cdots, c_{n}\right\}$ which satisfies the conditions (i), (ii), and (iii). Choose a generating set $\left\{1, b_{2}, \cdots, b_{q_{1}+1}, b_{q_{1}+2}, \cdots, b_{q_{1}+q_{2}+1}, \cdots, d_{n}\right\}$ with $b_{q_{1}+1}, \cdots, b_{q_{1}+q_{2}+1} \in J^{e-2}$. We continue choosing elements of our generating set in this way: At the $i$ th step we have already chosen $1, b_{2}, \cdots, b_{q_{i-1}+\cdots+q_{1}+1}$ and we suppose that the maximum number of elements in $J^{e-i}$ in any generating set satisfying (i), (ii), and (iii) which includes all of the above elements is $q_{2}$. We choose $b_{q_{i-1}+\cdots+q_{1}+2}, \cdots, b_{q_{i}+\cdots+q_{1}+1} \in J^{e-i}$ which are elements of some generating set satisfying the conditions of (i), (ii), 
and (iii) which also contain the elements we have chosen in the previous steps. After $e-1$ steps we have that $p, b_{2}, \cdots, b_{q_{e-1}+\cdots+q_{1}+1}$ generate all of $J$ and hence $1, b_{2}, \cdots, b_{n}$ is a generating set satisfying the conditions (i), (ii), and (iii). Also, this matrix representation depends on the order in which we index the $b_{i}$. We take $b_{1}=1$. We then assume that if $k_{i}$ is the order of $b_{i}$ that $k=k_{1} \geqq k_{2} \geqq \cdots \geqq k_{n}$. Next let $f_{i}$ be the largest positive integer such that $b_{i} \in J^{f_{i}}$. We call $f_{i}$ the radical index of $b_{i}$. We shall further assume that if $k_{i}=k_{j}$ with $i \geqq j$ then $f_{i} \geqq f_{j}$. We prove that the Szele representation of $R$ with respect to this ordering of the basis $b_{1}, \cdots, b_{n}$ of $R$ is of the desired type.

Again let $a \in R$ and write $b_{i} a=\sum_{j=1}^{n} \alpha_{i j} b_{j}$. Since we have that $k=k_{1} \geqq k_{2} \geqq \cdots \geqq k_{n}$ we already know that if $i>j$ with $k_{i}<k_{j}$ then $\alpha_{i j}$ is a multiple of $p^{k_{j}-k_{i}}$. So we need only restrict our attention to those $i, j$ such that $i>j$ but $k_{i}=k_{j}$. Let us express $a=\sum_{q=1}^{n} g_{q} b_{q}\left(g_{q} \in G_{k, r} q=1, \cdots, n\right)$. Then $b_{i} a=\sum_{q=1}^{n} b_{i} g_{q} b_{q}$. But by (ii), $b_{i} G_{k, r}=G_{k, r} b_{i}(i=1, \cdots, n)$ so $b_{i} g_{q} \in G_{k, r} b_{i}$. Let $b_{i} g_{q}=g_{q}^{(i)} b_{i}$. Then $b_{i} a=\sum_{q=1}^{n} g_{q}^{(i)} b_{i} b_{q}$. Next let

$$
b_{i} b_{q}=\sum_{j=1}^{n} \gamma_{i j}^{(q)} b_{j} \gamma_{i j}^{(q)} \in G_{k, r} \quad i, j, q=1, \cdots, n
$$

then

$$
b_{i} a=\sum_{j=1}^{n}\left(\sum_{q=1}^{n} g_{q}^{(i)} \gamma_{i j}^{(q)}\right) b_{j} .
$$

From this we conclude $\left(\alpha_{i j}\right) \dot{\phi}_{j}$, the $i, j$ th entry of the Szele representation of $a$ is $\left(\sum_{q=1}^{n} g_{q}^{(i)} \gamma_{i j}^{(q)}\right) \phi_{j}$. Thus if we seek to show that for an arbitrary $\left(\alpha_{i j}\right) \phi_{j}$ is a multiple of $p$ for some $i, j$ it suffices to show that $\gamma_{i j}^{(q)}$ is a multiple of $q$ for each $q=1, \cdots, n$.

We have thus reduced the problem to showing that if $i>j$ with $k_{i}=k_{j}$ then $\gamma_{i j}^{(q)}$ is a multiple of $p$ for each $q=1, \cdots, n$. If $q=1$ then $b_{i} b_{1}=b_{i}$ and so $\gamma_{i j}^{(1)}=\delta_{i j}$ and thus $\gamma_{i j}^{(1)}$ is a multiple of $p$ for all $i>j$. So the proof of the theorem will be complete if we can show that for all $q=2, \cdots, n \gamma_{i j}^{(q)}$ is a multiple of $p$ for all $i \geqq j$ such that $e_{i}=e_{j}$.

We shall assume that there is a $q \geqq 2$ for which there exists an $i \geqq j$ such that $e_{i}=e_{j}$ but $\gamma_{i j}^{(q)}$ is not a multiple of $p$, and we seek a contradiction. Since $q \geqq 2, b_{q} \in J$ so the radical index of $b_{i} b_{q}$ is strictly greater than $f_{i}$ which is the radical index of $b_{i}$ which is, by hypothesis, greater than or equal to the radical index of $\gamma_{j}$. So from the construction of the generating set $1, b_{2}, \cdots, b_{m}$ of $R$ over $G_{k, r}$ we will have our contradiction if we can show that $1, b_{2}, \cdots, b_{j-1}, b_{i} b_{q}, \cdots, b_{m}$ is a generating set of $R$ over $G_{k, r}$ satisfying the conditions (i), (ii), and (iii). 


$$
b_{\imath} b_{q}=\sum_{t=1}^{m} \gamma_{i t}^{(q)} b_{t}
$$

and $p$ does not divide $\gamma_{i j}^{(q)}$ so it follows that $1, b_{2}, \cdots, b_{j-1}, b_{i} b_{q}, b_{j+1}, \cdots, b_{m}$ is a generating set of $R$ over $G_{k, r}$. To show independence, we first note that $p^{k_{j}}\left(b_{i} b_{q}\right)=p^{k_{i}}\left(b_{i} b_{q}\right)=0$ so that order of $b_{\imath} b_{q}$ is less than or equal to $k_{j}$. However, $\gamma_{i j}^{(q)}$ is not a multiple of $p$ and so we conclude that $p^{s} b_{2} b_{q}=\sum_{t=1}^{m} \gamma_{i t}^{(q)} p^{s} b_{t} \neq 0$ if $s<k_{j}$ and thus the order of $b_{2} b_{q}$ is the order of $b_{j}$. Now since $1, b_{2}, \cdots, b_{j-1}, b_{i} b_{q}, b_{j+1}, \cdots, b_{m}$ is a generating set the map from the external direct sum

$$
\sum_{t=1}^{i-1} \oplus G_{k, r} b_{t} \oplus G_{k, r} b_{i} b_{q} \oplus \sum_{t=j+1}^{m} \oplus G_{k, r} b_{t}
$$

to $R$ given by

$$
\begin{gathered}
\left(g_{\imath}, g_{2} b_{2}, \cdots, g_{j-1} b_{j-1}, g_{j} b_{i} b_{q}, g_{j+1} b_{j+1}, \cdots, g_{m} b_{m}\right) \\
\longrightarrow \sum_{t=1}^{j-1} g_{t} b_{t}+g_{j} b_{i} b_{q}+\sum_{t=j+1}^{m} g_{t} b_{t}
\end{gathered}
$$

is onto. But as the order of $b_{i} b_{q}$ is $k_{j}$ we conclude that

$$
\sharp\left(\sum_{t=1}^{j-1} \oplus G_{k, r} b_{t} \oplus G_{k, r} b_{i} b_{q} \oplus \sum_{t=j+1}^{m} G_{k, r} b_{t}\right)=\# R
$$

which is equivalent to saying that $1, b_{2}, \cdots, b_{j-1}, b_{i} b_{q}, b_{j+1}, \cdots, b_{m}$ are independent. Moreover, if $g \in G_{k, r}$, then since $G_{k, r} b_{q}$ is a $\left(G_{k r}, G_{k, r}\right)$ submodule, there exists a $g^{\prime} \in G_{k_{r}}$ such that $b_{q} g=g^{\prime} b_{q}$. Similarly, given $g^{\prime}$ there exists a $g^{\prime \prime} \in G_{k, r}$ such that $b_{i} g^{\prime}=g^{\prime \prime} b_{i}$. Hence, $\left(b_{i} b_{q}\right) g=$ $g^{\prime \prime}\left(b_{i} b_{q}\right)$ and we conclude that $G_{k, r} b_{\imath} b_{q}$ is a $\left(G_{k, r}, G_{k, r}\right)$-submodule of $R$ and, therefore, $1, b_{2}, \cdots, b_{j-1}, b_{2} b_{q}, b_{\jmath+1}, \cdots, b_{m}$ satisfies the conditions of (i), (ii), and (iii) and the proof is complete.

As a result we obtain the following classification of finite nilpotent rings (of prime power order).

THEOREM 3.2. Let $R$ be a finite nilpotent ring of characteristic $p^{k}$. Then $R$ is isomorphic to a ring of Szele matrices (over $Z /(p)^{k}$ which are all strictly upper triangular modulo $p$.

Proof. We embed $R$ into the radical of a completely primary finite ring as follows. Let $\bar{R}=Z /\left(p^{k}\right)+R$ be the usual embedding of $R$ into a ring with 1 over $Z /\left(p^{k}\right) . \quad \bar{R}$ is completely primary because the nonunits of $\bar{R}$ are the elements of $p Z /\left(p^{k}\right)+R$ and bence form and ideal $J$ with $R \subset J . \quad \bar{R} \quad J . \quad \bar{R} / J \cong Z\left(p^{k}\right)$. We apply Theorem 3.1 to obtain Szele representation of $R$ which is upper triangular modulo $p$. The radical of $\bar{R}$ will be contained in the set of Szele matrices which are strictly upper traiangular modulo $p$ and the result follows. 
REMARK 3.3. In [4] Szele reduced the problem of classifying nilpotent Artinian rings to the problem of classifying finite nilpotent rings of prime power order by showing that any nilpotent Artinian ring is a direct sum or a particular type of extension of a finite nilpotent ring of prime power order by a direct sum of null rings over quasi-cycle groups. Thus Theorem 3.2 yields a classification of nilpotent Artinian rings which can by easily recovered by anyone with a knowledge of the results in [4].

REMARK 3.4. The question can be asked as to whether the author's previous representations [5] of completely primary and nilpotent finite rings are in fact the preimages under the map $\Phi$ defined in $\S 2$ of the Szele representation. The answer is no. Although the same generating sets were used in both representations (although they were ordered slightly differently) the author's previous representation is essentially the homological dual of the preimage of the Szele representation.

Note. The author would like to acknowledge a helpful remark by Professor K. R. McLean concerning Remark 3.3.

\section{REFERENCES}

1. G. J. Janusz, Separable algebras over commutative rings, Trans. Amer. Math. Soc., 122 (1966), 461-479.

2. R. Raghavendran, Finite associative rings, Compositio Math., 21 (1969), 195-229.

3. T. Szele, Ein Satz über die Struktur der endlichen Ringe, Acta Sci. Math., (Szeged)

11 (1948), 246-250.

4. - Nilpotent Artinian rings, Publ. Math. Debrecen, 4 (1955), 71-78.

5. R. S. Wilson, On the structure of finite rings, Compositio Math., 26 (1973), 79-93.

Received May 8, 1973 and in revised form July 15, 1973.

UNIVERSITY OF TEXAS 



\section{PACIFIC JOURNAL OF MATHEMATICS}

\section{EDITORS}

RICHARD ARENS (Managing Editor)

University of California

Los Angeles, California 90024

\section{J. DUGUNDJI}

Department of Mathematics University of Southern California Los Angeles, California 90007

D. Gilbarg and J. Milgram

Stanford University

Stanford, California 94305

University of Washington
Seattle, Washington 98105

ASSOCIATE EDITORS
E. F, BECKENBACH
B. H. NEUMANN
F. WOLF
K. Yoshida

\section{SUPPORTING INSTITUTIONS}

\author{
UNIVERSITY OF BRITISH COLUMBIA \\ CALIFORNIA INSTITUTE OF TECHNOLOGY \\ UNIVERSITY OF CALIFORNIA \\ MONTANA STATE UNIVERSITY \\ UNIVERSITY OF NEVADA \\ NEW MEXICO STATE UNIVERSITY \\ OREGON STATE UNIVERSITY \\ UNIVERSITY OF OREGON \\ OSAKA UNIVERSITY
}

\author{
UNIVERSITY OF SOUTHERN CALIFORNIA \\ STANFORD UNIVERSITY \\ UNIVERSITY OF TOKYO \\ UNIVERSITY OF UTAH \\ WASHINGTON STATE UNIVERSITY \\ UNIVERSITY OF WASHINGTON \\ * * * * \\ AMERICAN MATHEMATICAL SOCIETY \\ NAVAL WEAPONS CENTER
}

The Supporting Institutions listed above contribute to the cost of publication of this Journal, but they are not owners or publishers and have no responsibility for its content or policies.

Mathematical papers intended for publication in the Pacific Journal of Mathematics should be in typed form or offset-reproduced, (not dittoed), double spaced with large margins. Underline Greek letters in red, German in green, and script in blue. The first paragraph or two must be capable of being used separately as a synopsis of the entire paper. Items of the bibliography should not be cited there unless absolutely necessary, in which case they must be identified by author and Journal, rather than by item number. Manuscripts, in duplicate if possible, may be sent to any one of the four editors. Please classify according to the scheme of Math. Rev. Index to Vol. 39. All other communications to the editors should be addressed to the managing editor, or Elaine Barth, University of California, Los Angeles, California, 90024.

100 reprints are provided free for each article, only if page charges have been substantially paid. Additional copies may be obtained at cost in multiples of 50 .

The Pacific of Journal Mathematics is issued monthly as of January 1966. Regular subscription rate: $\$ 72.00$ a year (6 Vols., 12 issues). Special rate: $\$ 36.00$ a year to individual members of supporting institutions.

Subscriptions, orders for back numbers, and changes of address should be sent to Pacific Journal of Mathematics, 103 Highland Boulevard, Berkeley, California, 94708.

PUBLISHED BY PACIFIC JOURNAL OF MATHEMATICS, A NON-PROFIT CORPORATION

Printed at Kokusai Bunken Insatsusha (International Academic Printing Co., Ltd.), 270, 3-chome Totsuka-cho, Shinjuku-ku, Tokyo 160, Japan.

Copyright (C) 1973 by Pacific Journal of Mathematics Manufactured and first issued in Japan 


\section{Pacific Journal of Mathematics}

\section{Vol. 53, No. $2 \quad$ April, 1974}

Kenneth Abernethy, On characterizing certain classses of first countable spaces by

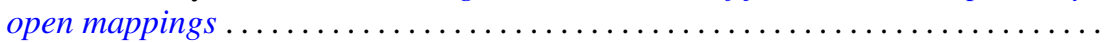

Ross A. Beaumont and Donald Lawver, Strongly semisimple abelian groups .......

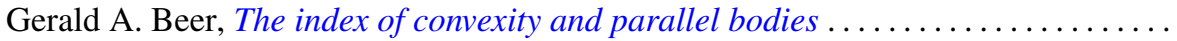

Victor P. Camillo and Kent Ralph Fuller, On Loewy length of rings ..............

Stephen LaVern Campbell, Linear operators for which $T^{*} T$ and $T T^{*}$ commute.

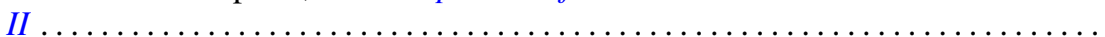

Charles Kam-Tai Chui and Philip Wesley Smith, Characterization of a function by

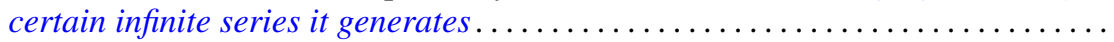

Allan L. Edelson, Conjugations on stably almost complex manifolds . ...........

Patrick John Fleury, Hollow modules and local endomorphism rings . . ..........

Jack Tilden Goodykoontz, Jr., Connectedness im kleinen and local connectedness in

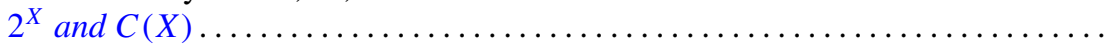

Robert Edward Jamison, II, Functional representation of algebraic intervals .......

Athanassios G. Kartsatos, Nonzero solutions to boundary value problems for

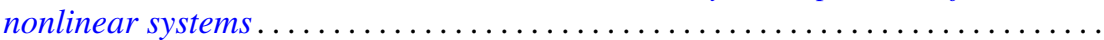

Soon-Kyu Kim, Dennis McGavran and Jingyal Pak, Torus group actions on simply

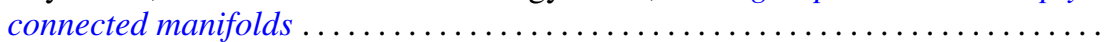

David Anthony Klarner and R. Rado, Arithmetic properties of certain recursively

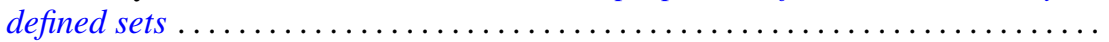

Ray Alden Kunze, On the Frobenius reciprocity theorem for square-integrable

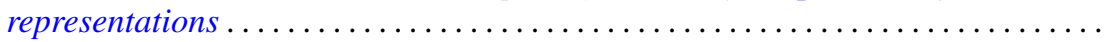

John Lagnese, Existence, uniqueness and limiting behavior of solutions of a class of differential equations in Banach space...

Teck Cheong Lim, A fixed point theorem for families on nonexpansive mappings Lewis Lum, A quasi order characterization of smooth continua

Andy R. Magid, Principal homogeneous spaces and Galois extensions . .

Charles Alan McCarthy, The norm of a certain derivation ..... . .

Louise Elizabeth Moser, On the impossibility of obtaining $S^{2} \times S^{1}$ by elementary surgery along a knot. .

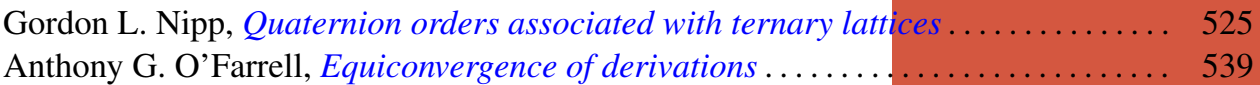

Dorte Olesen, Derivations of $A W^{*}$-algebras are inner . . . . . . . . . . . . . . . 555

Dorte Olesen and Gert Kjærgaard Pedersen, Derivations of $C^{*}$-algebras have

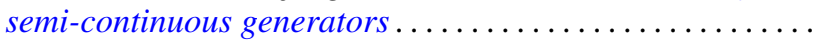

Duane O’Neill, On conjugation cobordism.

Chull Park and S. R. Paranjape, Probabilities of Wiener paths crossing differentiable

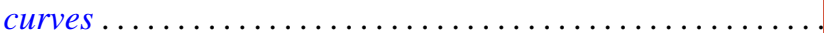

Edward Ralph Rozema, Almost Chebyshev subspaces of $L^{1}(\mu$;

Lesley Millman Sibner and Robert Jules Sibner, A note on the Atiyah-Bott fixed

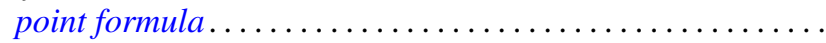

Betty Salzberg Stark, Irreducible subgroups of orthogonal groups generated by

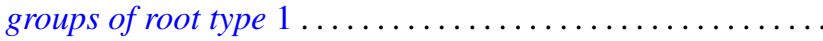

N. Stavrakas, A note on starshaped sets, $(k)$-extreme points and the half ray

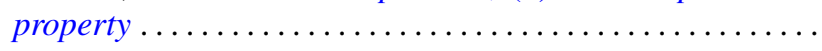

Carl E. Swenson, Direct sum subset decompositions of $Z \ldots \ldots$ 